\title{
Perturbed Sachdev-Ye-Kitaev Model: A Polaron in the Hyperbolic Plane
}

\author{
A. V. Lunkin, ${ }^{1,2,3}$ A. Yu. Kitaev, ${ }^{4}$ and M. V. Feigel'man $\oplus^{2,1}$ \\ ${ }^{1}$ Physics Department, National Research University "Higher School of Economics”, Moscow 101000, Russia \\ ${ }^{2}$ L. D. Landau Institute for Theoretical Physics, Kosygin street 2, Moscow 119334, Russia \\ ${ }^{3}$ Skolkovo Institute of Science and Technology, 143026 Skolkovo, Russia \\ ${ }^{4}$ California Institute of Technology, Pasadena, California 91125, USA
}

(Received 5 July 2020; accepted 2 October 2020; published 3 November 2020)

\begin{abstract}
We study the Sachdev-Ye-Kitaev $\left(\mathrm{SYK}_{4}\right)$ model with a weak $\mathrm{SYK}_{2}$ term of magnitude $\Gamma$ beyond the simplest perturbative limit considered previously. For intermediate values of the perturbation strength, $J / N \ll \Gamma \ll J / \sqrt{N}$, fluctuations of the Schwarzian mode are suppressed, and the $\mathrm{SYK}_{4}$ mean-field solution remains valid beyond the timescale $t_{0} \sim N / J$ up to $t_{*} \sim J / \Gamma^{2}$. The out-of-time-order correlation function displays at short time intervals exponential growth with maximal Lyapunov exponent $2 \pi T$, but its prefactor scales as $T$ at low temperatures $T \leq \Gamma$.
\end{abstract}

DOI: 10.1103/PhysRevLett.125.196602

Introduction.-The Sachdev-Ye-Kitaev (SYK) model [1-4] presents a rare example of an analytically tractable theory of strongly correlated fermions. While the original $\mathrm{SYK}_{4}$ model with only fourth-order terms in the Hamiltonian looks exotic from the condensed matter perspective, the inclusion of quadratic terms brings the theory closer to the world of strongly interacting electrons. Thus, the $\mathrm{SYK}_{4}$ Hamiltonian with a small $\mathrm{SYK}_{2}$ perturbation is a reasonable model in this regard. In the mean-field approximation (which is exact in the limit of infinite number of Majorana modes $N$ ), the $\mathrm{SYK}_{2}$ term is always dominant at temperature $T=0$ [5-10]. More exactly, the saddle point solution for the $\mathrm{SYK}_{4}$ Green's function, $G_{\mathrm{sp} 4}(t) \propto 1 / \sqrt{t}$, turns to $G_{\mathrm{sp} 2}(t) \propto 1 / t$ (characteristic of a Fermi liquid) at longer times. At finite $N$, the $\mathrm{SYK}_{4}$ phase is stable to quadratic perturbations below some threshold [11] due to soft mode fluctuations, which change the asymptotic behavior of Green's function to $G_{\text {fluc }}(t) \propto$ $1 / t^{3 / 2}$ at $t \gg t_{0} \sim N / J$ [4]. The stability result, originally obtained via straightforward perturbation theory [11], was later generalized to several related models (tunneling between different SYK grains, complex fermions) and to the renormalization group (RG) framework $[12,13]$. The threshold value of perturbation strength $\Gamma$ was estimated as $\Gamma_{c} \sim J / N$ and is more accurately determined below. If $\Gamma>\Gamma_{c}$, then the system behaves as a Fermi liquid at the longest times.

In the present Letter, we extend the results [11-13] in two important directions. First, we present a method to analyze the $\mathrm{SYK}_{4}+\mathrm{SYK}_{2}$ model beyond perturbation theory or its RG-like variant. Second, our new method works at a finite temperature (which is assumed to be greater than the temperature $T_{\mathrm{gl}}$ of an expected [14] glass transition). In particular, we study a previously unexplored range of intermediate strengths of the $\mathrm{SYK}_{2}$ term,
$J / N \ll \Gamma \ll J / \sqrt{N}$. In this regime, the quadratic perturbation is weak at the timescale $t \leq t_{0} \sim N / J$, where the saddle point (conformal) solution $G_{\mathrm{sp} 4}$ is applicable. Paradoxically, we find that the perturbation stabilizes the conformal solution $G_{\mathrm{sp} 4}(t) \sim 1 / \sqrt{t}$ for extended times, $t \gg t_{0}$, where Green's function of the pure $\mathrm{SYK}_{4}$ model is modified by the soft mode fluctuations. Only at the longest timescale, $t \geq J / \Gamma^{2} \gg t_{0}$, the conformal solution $G_{\mathrm{sp} 4}$ gives way to the Fermi-liquid solution $G_{\mathrm{sp} 2}(t) \propto 1 / t$.

Recent experiments on strongly correlated electron systems $[15,16]$ with nearly flat band demonstrate a "strange metal" behavior with resistivity $R(T) \propto T$, which was shown [8] (within purely saddle point analysis) to occur in an array of SYK "dots" coupled by weak tunneling. We demonstrate in this Letter that such a behavior can be realized under a much broader range of conditions than those implied in Ref. [8].

Our results are best understood using the geometric interpretation [17] of the Schwarzian theory in terms of an auxiliary particle whose trajectories are closed curves in the hyperbolic plane. The quadratic perturbation is then described as the particle being coupled to a free scalar Bose field. For sufficiently strong coupling, $\Gamma \gg J / N$, a polaron-type bound state is formed, resulting in increased rigidity of the curve and the suppression of its fluctuations.

The model and basic equations.-The Hamiltonian involves $N$ Majorana operators $\chi_{1}, \ldots, \chi_{N}$ and has the following form:

$$
H=\frac{1}{4 !} \sum_{i, j, k, l} J_{i j k l} \chi_{i} \chi_{j} \chi_{k} \chi_{l}+\frac{i}{2 !} \sum \Gamma_{i j} \chi_{i} \chi_{j} .
$$

Here $\left(J_{i j k l}\right)$ and $\left(\Gamma_{i j}\right)$ are antisymmetric tensors. Each of their components is a Gaussian random variable with zero mean and the following variance: $\overline{J_{i j k l}^{2}}=\left(3 ! J^{2} / N^{3}\right)$ and 
$\overline{\Gamma_{i j}^{2}}=\left(\Gamma^{2} / N\right)$. We assume that $\Gamma \ll J$. When averaging over disorder (i.e., over $J_{i j k l}$ and $\Gamma_{i j}$ ), Green's function is taken to be diagonal in replicas. This is self-consistent above the glass transition temperature $T_{\mathrm{gl}}$, where $\sqrt{N} \leq$ $\ln \left(J / T_{\mathrm{gl}}\right) \leq \frac{2}{3} s_{0} N$ with $s_{0}=0.4648 \ldots$, see [18]. We consider the problem in imaginary time, introduce new fields $G\left(\tau, \tau^{\prime}\right)$, and impose the constraint $G\left(\tau, \tau^{\prime}\right)=$ $-(1 / N) \sum_{i=1}^{N} \chi_{i}(\tau) \chi_{i}\left(\tau^{\prime}\right)$ using Lagrange multiplier fields $\Sigma\left(\tau, \tau^{\prime}\right)$. By performing the functional integral over the Grassmann variables $\chi_{i}$, we obtain an effective action for $G$ and $\Sigma$. The result has the form $S=S_{\mathrm{SYK}}+S_{2}$, where

$$
\begin{aligned}
\frac{2}{N} S_{\mathrm{SYK}}= & \operatorname{tr} \ln \left(-\partial_{\tau}-\Sigma\right) \\
+ & \int\left[\Sigma\left(\tau, \tau^{\prime}\right) G\left(\tau, \tau^{\prime}\right)-\frac{J^{2}}{4} G\left(\tau, \tau^{\prime}\right)^{4}\right] d \tau d \tau^{\prime}, \\
& \frac{2}{N} S_{2}=-\frac{\Gamma^{2}}{2} \int G\left(\tau, \tau^{\prime}\right)^{2} d \tau d \tau^{\prime} .
\end{aligned}
$$

The action $S_{\mathrm{SYK}}$ with the derivative term neglected - which is applicable for $t \gg 1 / J$-is invariant under reparametrizations of time. In particular, this abridged action has a degenerate saddle: along with a unique conformal [i.e., $\operatorname{PSL}(2, \mathbb{R})$-invariant] saddle point $\left(G_{c}, \Sigma_{c}\right)$, there is a manifold of related points $\left(G_{\varphi}, \Sigma_{\varphi}\right)$. It is parametrized by functions $\varphi$ that map the circle of length $\beta=1 / T$ to the circle of length $2 \pi$,

$$
\begin{aligned}
G_{\varphi}\left(\tau_{1}, \tau_{2}\right) & =J^{-2 \Delta} G_{c}\left(\varphi\left(\tau_{1}\right), \varphi\left(\tau_{2}\right)\right) \varphi^{\prime}\left(\tau_{1}\right)^{\Delta} \varphi^{\prime}\left(\tau_{2}\right)^{\Delta}, \\
G_{c}\left(\varphi_{1}, \varphi_{2}\right) & =-b^{\Delta} \operatorname{sgn}\left(\varphi_{12}\right)\left|\varphi_{12}\right|^{-2 \Delta},
\end{aligned}
$$

where $\Delta=\frac{1}{4}, b=(1 / 4 \pi)$, and $\varphi_{12}=2 \sin \left(\frac{\varphi_{1}-\varphi_{2}}{2}\right)$. (The corresponding expressions for $\Sigma$ differ by the replacement $\Delta \rightarrow 1-\Delta$ and an overall factor of $J^{2}$.) The term with $\partial_{\tau}$ in the original action (2) lifts the degeneracy and leads to the "Schwarzian action" defined on the soft mode manifold (4). Thus, the complete action (together with the quadratic term) becomes

$$
\begin{aligned}
S[\varphi]= & -\frac{\gamma}{J} \int_{0}^{\beta} \operatorname{Sch}\left(e^{i \varphi(\tau)}, \tau\right) d \tau \\
& -\frac{N \Gamma^{2}}{4} \int G_{\varphi}\left(\tau_{1}, \tau_{2}\right)^{2} d \tau_{1} d \tau_{2},
\end{aligned}
$$

where $\operatorname{Sch}(f(x), x)=\left[\left(f^{\prime \prime} / f^{\prime}\right)\right]^{\prime}-\frac{1}{2}\left[\left(f^{\prime \prime} / f^{\prime}\right)\right]^{2}$ is the Schwarzian derivative, $\gamma=\alpha_{S} N$, and $\alpha_{S} \sim 0.01$ is a numerical constant $[2,3]$; also see a comment on that matter in [11].

The use of action in the form of Eq. (5) needs some explanation. Although the second term in that equation is equal to $S_{2}$ defined by (3), the function $G_{\varphi}$ in it belongs to the soft mode manifold. In contrast, the $G$ in the original action $S_{\mathrm{SYK}}+S_{2}$ is completely general, so $S_{2}$ plays a dual role: (i) it competes with the main term $S_{\mathrm{SYK}}$ in determining the saddle point solution, and (ii) it reduces soft mode fluctuations since its very presence breaks down the reparametrization symmetry. The strength of the first effect is controlled by the parameter $\Gamma / J$ and does not depend on $N$. This is why, for small values of $\Gamma / J$, it is natural to start our analysis from effect (ii), which is captured by Eq. (5). We will see that the second term suppresses soft mode fluctuations and extends the domain where the $\mathrm{SYK}_{4}$ conformal solution is valid.

Geometric interpretation and the polaron analogy.The following picture is conceptually important, but we will do most calculations by a different method. Therefore, this section will be brief; more details can be found in the Supplemental Material [20]. The duality between the Schwarzian action and Jackiw-Teitelboim gravity was discussed in Ref. [17], as well as in the papers [21-23].

The geometric interpretation of the Schwarzian action [17] is based on a correspondence between functions $\varphi$ as described above and closed curves on the hyperbolic plane. Such curves may be parametrized by the proper length $\ell=J \tau$, which will be used instead of $\tau$ for the purpose of this discussion. In the Poincare disk model with metric $d s^{2}=\left(4 /\left(1-r^{2}\right)^{2}\right)\left(d r^{2}+r^{2} d \varphi^{2}\right)$, the curve is given by the equations $\varphi=\varphi(\ell)$ and $r=1-\varphi^{\prime}(\ell)$. This representation is valid if $\varphi^{\prime \prime}(\ell) \ll \varphi^{\prime}(\ell) \ll 1$, which is true for a typical curve of length $L=J \beta \gg 1$ in the statistical ensemble. Under the same conditions, we have $\operatorname{Sch}\left(e^{i \varphi(\ell)}, \ell\right)=K-1$, where $K$ is the extrinsic curvature of the curve at the given point. This allows for an elegant representation of the Schwarzian action $S_{\mathrm{Sch}}=$ $-\gamma \int_{0}^{L} \operatorname{Sch}\left(e^{i \varphi(\ell)}, \ell\right) d \ell$ in terms of the length of the curve and the enclosed area; however, some regularization is necessary in order to define the functional integral [17]. Replacing the function $\varphi$ with the curve $X$, we may rewrite Eq. (5) as follows:

$S[X]=S_{\mathrm{Sch}}[X]-\frac{N \Gamma^{2}}{4 J^{2}} \int G_{\Phi}^{2}\left(X\left(\ell_{1}\right), X\left(\ell_{2}\right)\right) d \ell_{1} d \ell_{2}$,

where $\quad G_{\Phi}\left(r_{1}, \varphi_{1} ; r_{2}, \varphi_{2}\right) \propto\left|\varphi_{12}\right|^{-2 \Delta}\left(1-r_{1}\right)^{\Delta}\left(1-r_{2}\right)^{\Delta}$ near the disk boundary. The function $G_{\Phi}$ can be identified with the propagator of a scalar boson $\Phi$. Thus, the nonlocal interaction between different points of the curve is decoupled, such that the action (6) is obtained from

$$
S[X, \Phi]=S_{\mathrm{Sch}}[X]+S_{\Phi}[\Phi]+\int_{0}^{L} \Phi(X(\ell)) d \ell,
$$

where $S_{\Phi}=(1 / 4 g \gamma) \int d \mu \Phi(x)\left(-\nabla^{2}-\frac{1}{4}+\delta^{2}\right) \Phi(x)$, by integrating out $\Phi$.

The action (7) is similar to the polaron problem, where an electron in a crystal interacts with an elastic deformation. By analogy with the heavy polaron, we will look for a mean-field solution where the field $\Phi$ forms a potential well 
close to the boundary of the Poincare disk. The general form of $\Phi$ in this region is $\Phi(r, \varphi)=\Lambda(\varphi)(1-r)^{\Delta}$, and the solution in question is $\Lambda(\varphi)=$ const. The curve roughly follows the circle $r=1-(2 \pi / L)$ and slightly wiggles. This behavior may be understood as a localized state of a quantum particle, whose coordinate is conveniently defined as $\xi=-\ln [\gamma(1-r)]$.

Adiabatic action.-We proceed with a formal solution for the polaron. It is convenient to rescale time as $\tau \rightarrow(J \tau / \gamma)$ and to introduce a similarly rescaled inverse temperature $\tilde{\beta}$ and a new coupling constant $g$,

$$
\tilde{\beta}=\frac{J \beta}{\gamma}, \quad g=\frac{b^{2 \Delta}}{2} \frac{N \Gamma^{2}}{J^{2}} \gamma^{2-4 \Delta}=\frac{N \gamma}{4 \sqrt{\pi}} \frac{\Gamma^{2}}{J^{2}} .
$$

Then the action (5) reads

$S[\varphi]=-\int_{0}^{\tilde{\beta}} \operatorname{Sch}\left(e^{i \varphi(\tau)}, \tau\right) d \tau-\frac{g}{2} \int\left(\frac{\varphi_{1}^{\prime} \varphi_{2}^{\prime}}{\varphi_{12}^{2}}\right)^{1 / 2} d \tau_{1} d \tau_{2}$.

Now we reduce the path integral with this action to some solvable quantum mechanical problem. To implement this idea, we introduce new time-dependent variables $\xi(\tau)=$ $-\ln \left[\varphi^{\prime}(\tau)\right]$ and $\Xi(\tau)=\left[\varphi^{\prime}(\tau)\right]^{1 / 2}$ and the corresponding Lagrange multipliers $\lambda(\tau)$ and $\Lambda(\tau)$. This means inserting $\delta\left(\varphi^{\prime}-e^{-\xi}\right)=\int_{-i \infty}^{+i \infty} \exp \left[\lambda\left(\varphi^{\prime}-e^{-\xi}\right)\right](d \lambda / 2 \pi i)$ and $\delta(\Xi-$ $e^{-\xi / 2}$ ) (expressed likewise using $\Lambda$ ) in the functional integral. Thus, the action takes the form

$$
S[\varphi, \xi, \lambda, \Xi, \Lambda]=\int_{0}^{\tilde{\beta}}\left(\frac{\xi^{\prime 2}}{2}-\lambda\left(\varphi^{\prime}-e^{-\xi}\right)-\frac{1}{2} e^{-2 \xi}-\Lambda\left(\Xi-e^{-\xi / 2}\right)\right) d \tau-\frac{g}{2} \iint \frac{\Xi\left(\tau_{1}\right) \Xi_{2}\left(\tau_{2}\right)}{\left|\varphi_{12}\right|} d \tau_{1} d \tau_{2} .
$$

We assume that $\tilde{\beta} \gg 1$ so that the term $\frac{1}{2} e^{-2 \xi}$ is relatively small. It will be neglected in our analysis.

We treat action (10) using adiabatic approximation, with $\xi$ being the fast variable. That is, the functional integral of $e^{-S}$ over $\xi$ is performed under the assumption that $\varphi^{\prime}(\tau)$, $\lambda(\tau), \Xi(\tau)$, and $\Lambda(\tau)$ are constant at a suitable timescale $\tau_{*}$ (to be determined later). The result has the form $e^{-S_{\text {eff }} \text {, }}$ where

$$
\begin{aligned}
S_{\mathrm{eff}}[\varphi, \lambda, \Xi, \Lambda]= & \int_{0}^{\tilde{\beta}}\left[E_{0}(\lambda, \Lambda)-\lambda \varphi^{\prime}-\Lambda \Xi\right] d \tau \\
& -\frac{g}{2} \int\left|\varphi_{12}\right|^{-1} \Xi\left(\tau_{1}\right) \Xi\left(\tau_{2}\right) d \tau_{1} d \tau_{2},
\end{aligned}
$$

and $E_{0}(\lambda, \Lambda)$ is the ground state of the effective Hamiltonian for the variable $\xi$,

$$
\hat{H}_{\lambda, \Lambda}=-\frac{1}{2} \partial_{\xi}^{2}+\Lambda e^{-\xi / 2}+\lambda e^{-\xi}
$$

This Hamiltonian has bound states with energies

$$
E_{n}=-\frac{(\kappa-1-2 n)^{2}}{32}, \quad n=0, \ldots,\left\lfloor\frac{\kappa-1}{2}\right\rfloor
$$

where $\kappa=-\sqrt{(8 / \lambda)} \Lambda$. The corresponding eigenfunctions $\psi_{n}(\xi)$ are provided in the Supplemental Material [20]. The characteristic time for the adiabatic approximation can be estimated as $\tau_{*} \sim\left(E_{1}-E_{0}\right)^{-1}=(8 / \kappa-2)$. Such an estimate is certainly correct for a harmonic oscillator, where the oscillation period is the only relevant timescale. The Hamiltonian (12) is similar if $\kappa \gg 1$. We will see that the last condition actually guarantees adiabaticity, i.e., that $\phi^{\prime}$, $\lambda, \Xi$, and $\Lambda$ do not fluctuate at the timescale $\tau_{*}$. In fact, the fluctuations at all timescales are small enough to be considered Gaussian.

Our next goal is to derive an effective action for $\varphi$. To this end, we find the saddle point of the action (11) with respect to the other variables. The saddle point conditions for $\lambda, \Lambda$, and $\Xi$ read

$$
\begin{gathered}
\varphi^{\prime}=\frac{\partial E_{0}}{\partial \lambda}=\frac{\kappa-1}{32} \frac{\kappa}{\lambda}, \quad \Xi=\frac{\partial E_{0}}{\partial \Lambda}=-\frac{\kappa-1}{16} \frac{\kappa}{\Lambda}, \\
\Lambda\left(\tau_{1}\right)=-g \int d \tau_{2} \frac{\Xi\left(\tau_{2}\right)}{\left|\varphi_{12}\right|} .
\end{gathered}
$$

Equations (14) allow one to eliminate $\lambda$ and $\Lambda$ from various formulas; in particular, the definition of $\kappa$ is equivalent to the relation $\Xi^{2}=\frac{\kappa-1}{\kappa} \varphi^{\prime}$. The integrand in the first term of the action (11) can be written as

$$
E_{0}(\lambda, \Lambda)-\lambda \varphi^{\prime}-\Lambda \Xi=\frac{\kappa-1}{32},
$$

and Eq. (15) becomes an equation for $\kappa(\tau)$,

$$
\kappa^{2}\left(\tau_{1}\right) \eta\left(\tau_{1}\right)=16 g \int d \tau_{2} \frac{\eta\left(\tau_{2}\right) \sqrt{\varphi^{\prime}\left(\tau_{1}\right) \varphi^{\prime}\left(\tau_{2}\right)}}{\left|\varphi_{12}\right|},
$$

where $\eta(\tau)=\sqrt{1-\kappa^{-1}(\tau)}$. Finally, the effective action is reduced to

$S=\int_{0}^{\tilde{\beta}} \frac{\kappa-1}{32} d \tau-\frac{g}{2} \int d \tau_{1} d \tau_{2} \frac{\eta\left(\tau_{1}\right) \eta\left(\tau_{2}\right) \sqrt{\varphi^{\prime}\left(\tau_{1}\right) \varphi^{\prime}\left(\tau_{2}\right)}}{\left|\varphi_{12}\right|}$.

Now, let $\kappa \gg 1$ so that $\eta(\tau) \approx 1$. Furthermore, we will assume (and later verify) that the fluctuations are small, and 
hence, both $\varphi^{\prime} \approx 2 \pi / \tilde{\beta}$ and $\kappa$ are nearly constant. Then Eq. (17) is simplified as follows:

$$
\kappa^{2}=16 g \int \frac{d \varphi\left(\tau_{2}\right)}{\left|2 \sin \left(\frac{\varphi\left(\tau_{1}\right)-\varphi\left(\tau_{2}\right)}{2}\right)\right|} \approx 32 g \ln \left(\frac{\kappa \tilde{\beta}}{16 \pi}\right),
$$

where we have used the cutoff $\left|\tau_{1}-\tau_{2}\right|>\tau_{*} \approx(8 / \kappa)$ for the logarithmic integral. As for the effective action (18), its first term may be neglected (see Supplemental Material [20]). Expressing $\varphi^{\prime}$ as a function of $\varphi$, namely, $\varphi^{\prime}(\tau)=\varepsilon(\varphi)$, we get

$$
S \approx-\frac{g}{2} \int_{0}^{2 \pi} \int_{0}^{2 \pi} \frac{d \varphi_{1}}{\varepsilon\left(\varphi_{1}\right)} \frac{d \varphi_{2}}{\varepsilon\left(\varphi_{2}\right)}\left(\frac{\varepsilon\left(\varphi_{1}\right) \varepsilon\left(\varphi_{2}\right)}{\varphi_{12}^{2}}\right)^{1 / 2} .
$$

Saddle point solution.-The action (20) attains its minimum at the constant field configuration, $\varepsilon(\varphi)=2 \pi / \tilde{\beta}$, as well as all configurations related to it by $\operatorname{PSL}(2, \mathbb{R})$ symmetries. The minimum value of the action, $S_{\min } \approx-\tilde{\beta} \kappa^{2} / 32$, determines a correction to the SYK free energy: $F \approx E_{0}-N s_{0} T-(J / \gamma)\left(\kappa^{2} / 32\right)$. Differentiating it and using Eq. (19), we find the entropy of the system

$$
S(T) \approx N s_{0}-\frac{g J}{\gamma T} .
$$

Note that the entropy vanishes at $T \sim \Gamma^{2} / J$, which is roughly the temperature at which the $\mathrm{SYK}_{4}$ conformal Green's function $G_{\mathrm{sp} 4}(\tau, 0) \sim-(J \tau)^{-1 / 2}$ gives way to the Fermi-liquid behavior, $G_{\mathrm{sp} 2}(\tau, 0) \sim-(\Gamma \tau)^{-1}$. At lower temperatures, $T \leq \Gamma^{2} / J$, we need to modify our polaron solution; namely, we should introduce an upper cutoff in Eq. (19), $\left|\tau_{1}-\tau_{2}\right|<J / \Gamma^{2}$. Thus, the equation for $\kappa$ becomes

$$
\kappa^{2}=32 g \ln \left(\frac{\kappa \bar{\beta}}{16 \pi}\right), \quad \bar{\beta}=\frac{J}{\gamma} \min \left(\beta, \frac{J}{\Gamma^{2}}\right) .
$$

Fluctuations.-Let us estimate the fluctuation of $\varepsilon(\varphi)$ around $\varepsilon_{0}=2 \pi / \tilde{\beta}$ and show that they are small. For simplicity, we assume that $T \gg \Gamma^{2} / J$. In addition to the adiabatic action $S$ given by Eq. (20), we need the first nonadiabatic correction. The latter is identical to the Schwarzian action (see Supplemental Material [20]). We consider the Fourier series $\varepsilon(\varphi)=\varepsilon_{0}+(1 / 2 \pi)$ $\sum_{n} \delta \varepsilon_{n} e^{i n \varphi}$, expand the effective action $S_{\text {Sch }}+S$ up to the second order in $\delta \varepsilon_{n}$ with $n \neq 0$, and calculate the Gaussian expectation values $\left\langle\delta \varepsilon_{n} \delta \varepsilon_{-n}\right\rangle$. (Note that $\delta \varepsilon_{0}$ is determined by the equation $\int(d \varphi / \varepsilon(\varphi))=\tilde{\beta}$.) This calculation, which can be found in the Supplemental Material [20], gives the following result:

$$
K_{\varepsilon}(n) \equiv \frac{\left\langle\delta \varepsilon_{n} \delta \varepsilon_{-n}\right\rangle}{\varepsilon_{0}^{2}}=\frac{2 \pi \varepsilon_{0}}{\varepsilon_{0}^{2}\left(n^{2}-1\right)+(g / 2) \tilde{\psi}(n)},
$$

where $\tilde{\psi}(n)=\psi\left(n+\frac{1}{2}\right)-\psi\left(-\frac{1}{2}\right)$ and $\psi(x)$ is the digamma function; thus, $\tilde{\psi}(n) \approx \ln (n)$ for $n \gg 1$. Equation (23) is accurate for $n \ll n_{*} \equiv\left(\varepsilon_{0} \tau_{*}\right)^{-1}=\kappa \tilde{\beta} /(16 \pi)$ because it was derived from the effective action that is valid at sufficiently long times, $\tau \gg \tau_{*}=8 / \kappa$. However, no inconsistency occurs at greater values of $n$ : for $n \gtrsim n_{*}$, the first term in the denominator of (23) starts to dominate over the second one, and the fluctuations are suppressed. The summation of the rhs of Eq. (23) over all $n$, or just those with $|n| \leq n_{*}$, leads to the estimate

$$
\frac{\left\langle(\delta \varepsilon)^{2}\right\rangle}{\varepsilon_{0}^{2}} \approx \frac{1}{\epsilon_{0} n_{*}}=\frac{8}{\kappa} \ll 1 .
$$

Thus, the fluctuations of $\varepsilon(\varphi)$ are much smaller than its typical value if $\kappa \gg 8$.

Phase diagram.-As parameter $\kappa$ decreases toward unity, the fluctuations become strong and the adiabatic approximation breaks down. At $\kappa \sim 1$, we expect a transition into another phase of our model, where the $\mathrm{SYK}_{2}$ term is irrelevant at all timescales [11]. In terms of the original parameters of the model, the transition occurs when $\Gamma$ becomes smaller than its critical value given by the equations

$$
\Gamma_{c} \sim \frac{J}{\sqrt{N \gamma \ln \frac{\bar{\beta}}{16 \pi}}}, \quad \bar{\beta}=\frac{J}{\gamma} \min \left(\frac{1}{T}, \frac{J}{\Gamma_{c}^{2}}\right),
$$

where $\gamma=\alpha_{S} N$. Note that the critical value $\Gamma_{c}=\Gamma_{c}(T)$ decreases logarithmically with the decrease of the physical temperature $T$, while $T \gtrsim\left(\Gamma_{c}^{2}(T) / J\right)$. At lower temperatures, $\Gamma_{c}(T)$ remains constant. The lines $\Gamma=\Gamma_{c}(T)$ and $T=J / \gamma$ (see Fig. 1) separate the region with strong fluctuations, characterized by Green's function $G_{\text {fluc }}(\tau, 0) \sim$ $\gamma(J \tau)^{-3 / 2}$ for sufficiently large $\tau$ (but still much less than $\beta$ ), from regions where the saddle point solution is valid.

Higher-order Green's functions.-Conformal solution (4) describes single-particle fermionic Green's function of

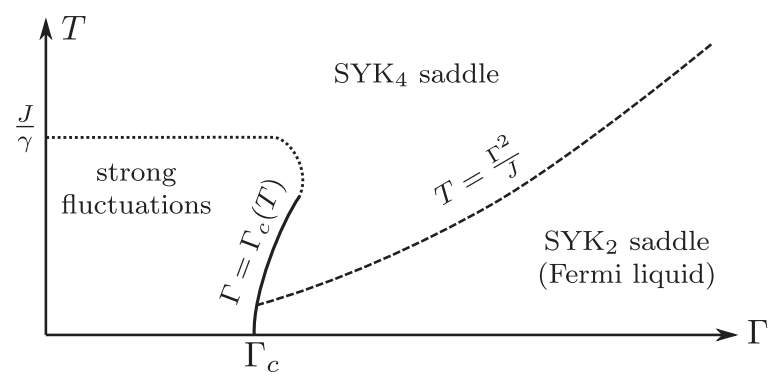

FIG. 1. Regions with different Green's function behaviors at large $\tau$. The phase boundaries are not sharp, except for the boundary between the fluctuation region and saddle point regions (the solid line). The latter is well defined asymptotically, under the condition $T \ll J / \gamma$. Hence, the termination point of the solid line, given by the condition $T \sim J / \gamma$, is fuzzy. 
the original problem with Hamiltonian (1). Additional information on its quantum dynamics is provided by higher-order fermion Green's functions defined as $G^{(p)}\left(\tau, \tau^{\prime}\right) \equiv\left[-(1 / N) \sum_{i} \chi_{i}(\tau) \chi_{i}\left(\tau^{\prime}\right)\right]^{p}$. It can be shown (see Supplemental Material, Sec. III [20]) that the functions $G^{(p)}\left(\tau, \tau^{\prime}\right)$ with $p \ll \kappa$ can be calculated by means of the effective action (20) and its propagator (23). To find them, we need just to average $p$ power of the conformal solution (4) over fluctuations of variables $\xi$ and $\varphi$ described by the polaron bound state:
$G^{(p)}\left(\tau_{1}, \tau_{2}\right)=(-1)^{p}\left\langle\left\{b e^{-\xi_{1}-\xi_{2}} \sin ^{-2}\left[\frac{1}{2}\left(\varphi_{1}-\varphi_{2}\right)\right]\right\}^{p / 4}\right\rangle$. The result of calculations (provided in the Supplemental Material, Sec. III [20]) reads (remember that $\kappa \ll N$ )

$$
\frac{G^{(p)}\left(\tau_{1}-\tau_{2}\right)}{\left[G\left(\tau_{1}-\tau_{2}\right)\right]^{p}}=\exp \left(\frac{p^{2}}{4 \kappa}\left[1+f\left(\theta_{12}\right)\right]\right)
$$

where $\theta_{12}=2 \pi T\left(\tau_{1}-\tau_{2}\right)$ and function $f(\theta)$ is provided below $\left(n_{*} \varepsilon_{0}=\kappa / 8\right)$,

$$
f(\theta)=\frac{2+n_{*} \theta}{\left(n_{*} \theta\right)^{2}}\left[2 n_{*} \theta \cosh \left(\frac{n_{*} \theta}{2}\right)-4 \sinh \left(\frac{n_{*} \theta}{2}\right)\right] \exp \left\{-\frac{n_{*} \theta}{2}\right\}=\left\{\begin{array}{cc}
1 & n_{*} \theta \gg 1 \\
\frac{\theta n_{*}}{3} & n_{*} \theta \ll 1
\end{array}\right. \text {. }
$$

Two terms in the exponent of Eq. (26) come from the averaging over fluctuations of $\xi_{1,2}$ (first term) and angular variables $\varphi_{1,2}$.

Out-of-time-order correlator (OTOC).-The OTOC is defined as the following irreducible average:

$$
\mathcal{F}\left(\theta_{1}, \theta_{2} ; \theta_{3}, \theta_{4}\right)=\left\langle\left\langle G\left(\theta_{1}, \theta_{2}\right) G\left(\theta_{3}, \theta_{4}\right)\right\rangle\right\rangle,
$$

where $\langle\ldots\rangle$ means averaging with respect to fluctuations of $\delta \varepsilon(\varphi)$. We assume that $\operatorname{Re} \theta_{3}>\operatorname{Re} \theta_{1}>\operatorname{Re} \theta_{4}>\operatorname{Re} \theta_{2}$. The function $\mathcal{F}$ depends, in general, on four independent variables, but here we consider the special case $\theta_{1}=(\pi-\theta / 2), \quad \theta_{2}=(-\pi-\theta / 2), \quad \theta_{3}=(\pi+\theta / 2)$, $\theta_{4}=(-\pi+\theta / 2)$; see, for example, Ref. [24]. It is convenient to introduce the function $f(\theta)=$ $\left[\left(\mathcal{F}\left(\theta_{1}, \theta_{2} ; \theta_{3}, \theta_{4}\right)\right) /\left(G\left(\theta_{1}, \theta_{2}\right) G\left(\theta_{3}, \theta_{4}\right)\right)\right]$; we calculate its major term of the order of $O(1 / N)$ in the limit $N \gg 1$. After the calculation of $f(\theta)$ within the imaginary time technique, we need to perform analytical continuation to real times by the substitution $f\left(\theta \rightarrow-i \varepsilon_{0} t\right) \equiv F(t)$. Exponential growth of $F(t)$ at short times demonstrate quantum-chaotic behavior of the system.

To calculate $f(\theta)$, we first write $G\left(\theta_{1}, \theta_{2}\right)=$ $\left\langle G\left(\theta_{1}, \theta_{2}\right)\right\rangle+\delta G\left(\theta_{1}, \theta_{2}\right)$, where

$$
\begin{aligned}
\frac{\delta G\left(\theta_{1}, \theta_{2}\right)}{G\left(\theta_{1}, \theta_{2}\right)} & =\frac{1}{2 \pi} \sum_{m \neq 0, \pm 1}\left[i m e^{i m \theta_{2}}+i m e^{i m \theta_{1}}\right. \\
& \left.+\cot \left(\frac{\theta_{1}-\theta_{2}}{2}\right)\left(e^{i m \theta_{2}}-e^{i m \theta_{1}}\right)\right] \frac{\varepsilon_{m}}{i m \varepsilon_{0}}
\end{aligned}
$$

The same expansion is used for $G\left(\theta_{3}, \theta_{4}\right)$, and the results are substituted into Eq. (28), leading to the following Fourier series:

$$
\begin{aligned}
f(\theta) & =\frac{1}{(2 \pi)^{2}} \sum_{m \neq 0, \pm 1} 4 \cos ^{2}\left(\frac{m \pi}{2}\right) \cos (m \theta) K_{\varepsilon}(m) \\
& =\frac{1}{\pi^{2}} \sum_{n \neq 0} e^{2 i n \theta} K_{\varepsilon}(2 n),
\end{aligned}
$$

where $K_{\varepsilon}(m)$ is provided by Eq. (23). The series in (30) can be calculated by the transformation to the integral over $d n$, which is determined (after analytic continuation to real $t$ and in the limit $t \gg 1 / T$ ) by the contribution of the single pole of $K_{\varepsilon}(2 n)$ at $n=\frac{1}{2}$. Finally, we obtain

$$
F(t) \approx i \frac{\varepsilon_{0} e^{2 \pi T t}}{2 \varepsilon_{0}^{2}+g\left(\frac{\pi^{2}}{4}-2\right)},
$$

with $\varepsilon_{0}=2 \pi / \tilde{\beta} \equiv 2 \pi \gamma T / J$. The Lyapunov exponent $\lambda=2 \pi T$ is the same as in the pure $\mathrm{SYK}_{4}$ model, but the preexponential factor is considerably modified, as the second term in denominator of Eq. (31) dominates at $T \ll \Gamma$, where $F(t)=(i / N)\left(T / T_{*}\right) e^{2 \pi T t}$ and $T_{*}=\Gamma^{2} / J$.

Conclusions. - We have shown that a moderate quadratic perturbation to the $\mathrm{SYK}_{4}$ model with $N$ Majorana modes can be described in terms of a self-consistent polaron-type solution. The presence of such a perturbation with strength $\Gamma$ in the interval $J / N \leq \Gamma \leq J / \sqrt{N}$ stabilizes the conformal saddle point solution for the Majorana Green's function within a broad range of energies and temperatures. The $\mathrm{SYK}_{4}$ mean-field Green's function $G(\epsilon) \sim 1 / \sqrt{J \epsilon}$ [defined at Matsubara frequencies $\left.\epsilon=i 2 \pi T\left(n+\frac{1}{2}\right)\right]$ is valid down to $T_{*} \sim \Gamma^{2} / J$, where a crossover to a Fermi liquid at $\epsilon \lesssim T_{*}$ occurs. A schematic "phase diagram" of the model is shown in Fig. 1. At low temperatures, there is a genuine phase transition at $\Gamma=\Gamma_{c}$, where $\Gamma_{c}$ is defined in (25). Specifically at $T=0$, the $\epsilon \rightarrow+0$ asymptotics change from $G(\epsilon) \propto \sqrt{\epsilon}$ for $\Gamma<\Gamma_{c}$ to $G(\epsilon) \simeq 1 / \Gamma$ for $\Gamma>\Gamma_{c}$. Note that higher-order Green's functions $G^{(p)}(\tau)$ display exponential growth with $p$, Eq. (26). The prefactor of the OTOC function (31) is proportional to $T$ in a low temperature 
range, $T_{*}<T \ll \Gamma$, indicating slightly less chaotic behavior of the system due to the presence of quadratic perturbations. These results can be relevant to the description of strongly correlated electron systems with flat bands of various origin $[15,16,25,26]$.

M. V.F. is grateful to L. B. Ioffe, A. Kamenev, V.E. Kravtsov, and K. S. Tikhonov for useful discussions. Research of A. V.L. was partially supported by the Foundation for Advancement of Theoretical Physics and Mathematics "Basis" and Basic research program of HSE. A. K. is supported by the Simons Foundation under Grant No. 376205 and through the "It from Qubit" program, as well as by the Institute of Quantum Information and Matter, a NSF Frontier center funded in part by the Gordon and Betty Moore Foundation.

[1] Proceedings of the KITP (2015), http://online.kitp.ucsb.edu/ online/entangled15/kitaev/, http://online.kitp.ucsb.edu/ online/entangled15/kitaev2/.

[2] A. Kitaev and S. J. Suh, J. High Energy Phys. 05 (2018) 183.

[3] J. Maldacena and D. Stanford, Phys. Rev. D 94, 106002 (2016).

[4] D. Bagrets, A. Altland, and A. Kamenev, Nucl. Phys. B911, 191 (2016).

[5] S. Banerjee and E. Altman, Phys. Rev. B 95, 134302 (2017).

[6] R. A. Davison, W. Fu, A. Georges, Y. Gu, K. Jensen, and S. Sachdev, Phys. Rev. B 95, 155131 (2017).

[7] S.-K. Jian and H. Yao, Phys. Rev. Lett. 119, 206602 (2017).

[8] X.-Y. Song, C.-M. Jian, and L. Balents, Phys. Rev. Lett. 119, 216601 (2017).

[9] P. Zhang, Phys. Rev. B 96, 205138 (2017).

[10] A. Haldar, S. Banerjee, and V. B. Shenoy, Phys. Rev. B 97, 241106(R) (2018).
[11] A. V. Lunkin, K. S. Tikhonov, and M. V. Feigelman, Phys. Rev. Lett. 121, 236601 (2018).

[12] A. Altland, D. Bagrets, and A. Kamenev, Phys. Rev. Lett. 123, 106601 (2019).

[13] A. Altland, D. Bagrets, and A. Kamenev, Phys. Rev. Lett. 123, 226801 (2019).

[14] A. Georges, O. Parcollet, and S. Sachdev, Phys. Rev. B 63, 134406 (2001).

[15] Y. Cao, D. Chowdhury, D. Rodan-Legrain, O. RubiesBigorda, K. Watanabe, T. Taniguchi, T. Senthil, and P. Jarillo-Herrero, Phys. Rev. Lett. 124, 076801 (2020).

[16] H. Polshyn, M. Yankowitz, S. Chen, Y. Zhang, K. Watanabe, T. Taniguchi, C. R. Dean, and A.F. Young, Nat. Phys. 15, 1011 (2019).

[17] A. Kitaev and S. J. Suh, J. High Energy Phys. 05 (2019) 198.

[18] Left estimate follows from the result of Ref. [14], which was criticized in Ref. [19]; right estimate corresponds to the temperature where entropy of the replica-symmetric solution of $\mathrm{SYK}_{4}$ model vanishes.

[19] G. Gur-Ari, R. Mahajana, and A. Vaezi, J. High Energy Phys. 11 (2018) 070.

[20] See Supplemental Material at http://link.aps.org/ supplemental/10.1103/PhysRevLett.125.196602for technical details.

[21] K. Jensen, Phys. Rev. Lett. 117, 111601 (2016).

[22] J. Maldacena, D. Stanford, and Z. Yang, Prog. Theor. Exp. Phys. (2016), 12C104.

[23] J. Engelsöy, T. G. Mertens, and H. Verlinde, J. High Energy Phys. 07 (2016) 139.

[24] D. Bagrets, A. Altland, and A. Kamenev, Nucl. Phys. B921, 727 (2017).

[25] A. Chen, R. Ilan, F. de Juan, D. I. Pikulin, and M. Franz, Phys. Rev. Lett. 121, 036403 (2018).

[26] N. J. G. Couto, B. Sacépé, and A. F. Morpurgo, Phys. Rev. Lett. 107, 225501 (2011). 